\title{
UNIFORM BOUNDS FOR STRONGLY F-REGULAR SURFACES
}

\author{
PAOLO CASCINI, YOSHINORI GONGYO, AND KARL SCHWEDE
}

\begin{abstract}
We show that if $(X, B)$ is a two dimensional Kawamata log terminal pair defined over an algebraically closed field of characteristic $p$, and $p$ is sufficiently large, depending only on the coefficients of $B$, then $(X, B)$ is also strongly $F$-regular.
\end{abstract}

\section{INTRODUCTION}

It has been well understood for a long time that in the study of birational geometry over the complex field, it is convenient to work with log pairs with mild singularities, such as Kawamata log terminal singularities. On the other hand, in the study of birational geometry in positive characteristic, it is not clear what is the right category of singularities. On one hand, Kawamata log terminal singularities are the right singularities because they are preserved by the minimal model program and, on the other hand, strongly $F$-regular singularities, which are defined via the Frobenius morphism, are important because they allow one to extend many results known over the complex field to an algebraically closed field of positive characteristic.

The aim of this paper is to study the relationship within these two categories of singularities in dimension two. In a sequence of papers, Hara-Watanabe [7, Hara-Yoshida [8, and Takagi [19] proved that if $(X, B)$ is a log pair defined over the complex numbers, then $(X, B)$ is Kawamata log terminal if and only if its reduction modulo $p$ is strongly $F$-regular, for any sufficiently large $p$. In addition, any strongly $F$-regular pair is always Kawamata log terminal. On the other hand, even in the case of surfaces, it is possible to show the existence of Kawamata log terminal pairs which are not strongly $F$-regular (e.g. see Example 3.4 for a sequence of examples in any characteristic).

Here we prove that if $(X, B)$ is a two dimensional Kawamata log terminal pair defined over an algebraically closed field of characteristic $p$, and $p$ is sufficiently large, depending on the coefficients of $B$, then $(X, B)$ is also strongly $F$-regular.

More specifically, our main theorem is the following:

Theorem 1.1. Let $I \subseteq(0,1) \cap \mathbb{Q}$ be a finite subset and let $\Gamma=D(I)$ (cf. Definition 2.1). Then there exists a positive constant $p_{0}$ depending only on $I$ such that if

Received by the editors February 15, 2014 and, in revised form, July 10, 2014.

2010 Mathematics Subject Classification. Primary 14F18, 13A35, 14 B05.

Key words and phrases. F-regular, F-pure, log terminal, log canonical.

The first author was partially supported by EPSRC grant P28327.

The second author was partially supported by the Grand-in-Aid for Research Activity Start-Up $\sharp 24840009$ from JSPS and research expense from the JRF fund.

The third author was partially supported by the NSF grant DMS \#1064485, NSF FRG grant DMS \#1265261/1501102, NSF CAREER grant DMS \#1252860/1501115 and a Sloan Fellowship. 
$\left(X, B:=\sum_{i=1}^{\ell} q_{i} D_{i}\right)$ is a two dimensional Kawamata log terminal pair defined over an algebraically closed field of characteristic $p>p_{0}$ and such that the coefficients of $B$ belong to $\Gamma$, then $(X, B)$ is strongly $F$-regular.

Note that in the absolute case, i.e. assuming that $B=0$, then the result follows from [5], with $p_{0}=5$. For an arbitrary finite set $I$, it is possible to find $p_{0}$ effectively, using the bound of Corollary [3.9, although this bound is not sharp.

Thanks to the work of Hacon and $\mathrm{Xu}$ [4, we are able to show the existence of dlt flips, if the characteristic of the underlying field is sufficiently large:

Theorem 1.2. Let $I \subseteq(0,1]$ be a finite set. Then there exists a prime $p_{I}$, depending only on $I$, such that if $(X, B)$ is a three dimensional dlt pair over an algebraically closed field of characteristic $p>p_{I}$, such that the coefficients of $B$ belong to $I$, and $f: X \rightarrow Y$ is a $(X, B)$-flipping contraction, then the flips exist.

Recently Birkar 2] has proven a stronger version of the result above by showing that dlt flips always exist over any algebraically closed field of characteristic $p_{I}>5$, by using different methods than ours.

The paper is organised as follows: In Section 2, we introduce the tools used in the rest of the paper. In Section 3, we prove Theorem 1.1 in the special case $X=\mathbb{A}^{2}$ and where $B$ is a line arrangement. By a standard cone construction, this allows us to show that if $\left(\mathbb{P}^{1}, B\right)$ is a log Fano pair defined over a field of characteristic $p$ and $p$ is sufficiently large, depending on the coefficients of $B$, then $\left(\mathbb{P}^{1}, B\right)$ is globally $F$-regular. Thus, by using a global to local method, this leads to a proof of Theorem 1.1] in the general case, as in Section 4. Finally, in Section 5, we prove Theorem 1.2

\section{Preliminary Results}

We work over an algebraically closed field $k$ of positive characteristic $p$, unless otherwise stated.

We refer to [10] for the classical definitions of singularities appearing in the minimal model program (e.g. Kawamata log terminal pairs), except for the fact that in our definitions we require the pairs to have effective boundaries. Given a $\log$ pair $(X, \Delta)$ and a geometric valuation $E$ over $X$, we denote by $a(E, X, \Delta)$ the $\log$ discrepancy of $(X, \Delta)$ with respect to $E$. We say that a pair $(X, \Delta)$ is $\log$ Fano if it is Kawamata log terminal and $-\left(K_{X}+\Delta\right)$ is ample.

We refer to [16] for the classical definition of singularities in positive characteristic (see also Definition 2.4 and Definition 2.7).

Given a subset $I \subseteq[0,1]$, we will say that $I$ is $A C C$ (respectively $D C C$ ) if it satisfies the ascending chain condition (respectively the descending chain condition).

Definition 2.1 (Hyperstandard set). Let $I \subseteq[0,1]$ be a subset. We define

$$
I_{+}=\left\{\sum_{j=1}^{m} a_{j} i_{j} \mid i_{j} \in I, a_{j} \in \mathbb{N} \text { for } j=1, \ldots, m\right\} \cap[0,1]
$$

and

$$
D(I)=\left\{\frac{m-1+f}{m} \mid m \in \mathbb{N}, f \in I_{+}\right\} \cap[0,1] .
$$


The following results are well known.

Lemma 2.2 ([11, Lemma 4.4]). Let $I \subseteq[0,1]$ be a subset. Then

$$
D(D(I))=D(I) \cup\{1\}
$$

and $I$ is $D C C$ if and only if $D(I)$ is $D C C$.

Lemma 2.3 ([11, Lemma 4.3]). Let $(X, \Delta)$ be a log canonical pair such that the components of $\Delta$ belong to a subset $I \subseteq[0,1]$, and let $S$ be an irreducible component of $\lfloor\Delta\rfloor$. Let $\Theta$ be the divisor on $S$ defined by adjunction:

$$
\left.\left(K_{X}+\Delta\right)\right|_{S}=K_{S}+\Theta .
$$

Then, the coefficients of $\Theta$ belong to $D(I)$.

2.1. Some remarks on $F$-pure thresholds. Suppose that $D \geq 0$ is a divisor on a normal integral affine scheme $X=\operatorname{Spec} R$. We begin by recalling the definition of sharp $F$-purity and the $F$-pure threshold.

Definition 2.4 (Sharp $F$-purity and strong $F$-regularity). For any real number $\lambda \geq 0$, we say that $(X, \lambda D)$ is sharply $F$-pure if for some $e>0$ there exists

$$
\phi \in \operatorname{Hom}_{\mathcal{O}_{X}}\left(F_{*}^{e}\left(\mathcal{O}_{X}\left(\left\lceil\left(p^{e}-1\right) \lambda D\right\rceil\right)\right), \mathcal{O}_{X}\right)
$$

such that $\phi\left(F_{*}^{e} \mathcal{O}_{X}\right)=\mathcal{O}_{X}$.

We say that $(X, \lambda D)$ is strongly $F$-regular if for every effective Weil divisor $E \geq 0$ there exists $e>0$ and

$$
\phi \in \operatorname{Hom}_{\mathcal{O}_{X}}\left(F_{*}^{e}\left(\mathcal{O}_{X}\left(\left\lceil\left(p^{e}-1\right) \lambda D+E\right\rceil\right)\right), \mathcal{O}_{X}\right)
$$

such that $\phi\left(F_{*}^{e} \mathcal{O}_{X}\right)=\mathcal{O}_{X}$.

Remark 2.5. In the above definition of sharp $F$-purity, if a single $e>0$ yields a $\phi$, then all multiples $n e$ of that $e>0$ also yield elements

$$
\phi^{n} \in \operatorname{Hom}_{\mathcal{O}_{X}}\left(F_{*}^{n e}\left(\mathcal{O}_{X}\left(\left\lceil\left(p^{n e}-1\right) \lambda D\right\rceil\right)\right), \mathcal{O}_{X}\right)
$$

such that $\phi^{n}\left(F_{*}^{n e} \mathcal{O}_{X}\right)=\mathcal{O}_{X}$.

Definition 2.6 (F-pure threshold). With $(X, D)$ as above, the $F$-pure threshold of $(X, D)$, denoted $\operatorname{fpt}(X, D)$, is defined to be

$$
\sup \{t>0 \mid(X, t D) \text { is sharply } F \text {-pure }\} \text {. }
$$

In the case that $X$ is $\mathbb{Q}$-Gorenstein and $D$ is $\mathbb{Q}$-Cartier, it can be shown that $\operatorname{fpt}(X, D)$ is a rational number $[17$.

2.2. Globally $F$-regular pairs. We now recall the definition of globally $F$-regular pairs:

Definition 2.7 (Global $F$-regularity; 4, 16, 18). Let $f: X \rightarrow Y$ be a proper morphism of normal varieties and let $\Delta \geq 0$ be a $\mathbb{Q}$-divisor on $X$. Then $(X, \Delta)$ is globally F-regular over $Y$ if for any effective divisor $D$, there exists a positive integer $e$ such that

$$
\mathcal{O}_{X} \rightarrow \mathcal{O}_{X}\left(\left\lceil\left(p^{e}-1\right) \Delta\right\rceil+D\right)
$$

splits locally over $Y$. 
Remark 2.8. Using the same notation as Definition 2.7 if $Y$ is affine, then $(X, \Delta)$ is globally $F$-regular over $Y$ if and only if for any effective divisor $D$, there exists a positive integer $e$ such that the natural map

$$
H^{0}\left(X, \mathcal{O}_{X}\left(\left\lfloor\left(1-p^{e}\right)\left(K_{X}+\Delta\right)\right\rfloor-D\right)\right) \rightarrow H^{0}\left(X, \mathcal{O}_{X}\right)
$$

is surjective [4, Proposition 2.10].

Let $X$ be a normal projective variety and let $L$ be an ample divisor on $X$. We denote by $R(X, L)=\bigoplus_{m \in \mathbb{Z}} H^{0}\left(X, \mathcal{O}_{X}(m L)\right)$ the section ring of $L$. The corresponding affine cone over $X$ is given by

$$
W=\operatorname{Spec} R(X, L) .
$$

For each effective $\mathbb{Q}$-divisor $\Delta$ on $X$, we denote by $\Delta_{W}$ the associated $\mathbb{Q}$-divisor on $W$ (e.g. see [9, §3.1] for more details).

Proposition 2.9. Let $X$ be a normal variety, $\Delta \geq 0$ a $\mathbb{Q}$-divisor on $X$ and $L$ an ample divisor. Let $W$ be the affine cone over $X$ associated to $L$ and let $\Delta_{W}$ be the corresponding $\mathbb{Q}$-divisor.

Then $K_{W}+\Delta_{W}$ is $\mathbb{Q}$-Cartier if and only if $r\left(K_{X}+\Delta\right) \sim_{\mathbb{Q}} L$ for some rational number $r \in \mathbb{Q}$. In this case:

(1) $(X, \Delta)$ is log Fano if and only if $\left(W, \Delta_{W}\right)$ is Kawamata log terminal.

(2) $(X, \Delta)$ is globally $F$-regular if and only if $\left(W, \Delta_{W}\right)$ is strongly $F$-regular (in fact, this holds even without the $\mathbb{Q}$-Cartier assumption).

Proof. (1) is well known (e.g. see [9, Lemma 3.1]). (2) follows from [16, Proposition $5.3]$

Lemma 2.10. Let $(X, \Delta)$ be a sharply $F$-pure pair and let $C$ be an effective divisor such that $(X, \Delta)$ is strongly $F$-regular outside the support of $C$ and $(X, \Delta+\varepsilon C)$ is sharply $F$-pure for some $\varepsilon>0$. Then $(X, \Delta)$ is strongly $F$-regular.

Proof. We may assume that $X$ is affine. Thus, the claim follows from [16, Corollary 3.10].

The following result is a slight generalization of [5, Proposition 4.3] and [4, Proposition 3.8].

Proposition 2.11. Let $f: X \rightarrow Y$ be a birational morphism of normal varieties. Let $\Delta$ and $B$ be $\mathbb{Q}$-divisors on $X$ such that $(X, \Delta)$ is purely log terminal, $S=\lfloor\Delta\rfloor$ is prime and normal, $(X, B)$ is Kawamata log terminal and $B+S \leq \Delta$. Assume that

(1) $-\left(K_{X}+\Delta\right)$ is f-ample,

(2) $S$ is $f$-exceptional,

(3) if $K_{S}+\Delta_{S}=\left.\left(K_{X}+\Delta\right)\right|_{S}$ is defined by adjunction, then $\left(S, \Delta_{S}\right)$ is a globally F-regular pair.

Then $(X, B)$ is globally $F$-regular over $Y$.

Proof. By standard perturbation techniques (e.g. see [4, Lemma 2.8 and 2.13]), we may assume that the $\mathbb{Q}$-Cartier indices of $K_{X}+\Delta$ and $K_{X}+B$ are not divisible by $p$. We may also assume that $Y$ is affine. Let $E$ be an effective divisor on $X$. We may write $E=n_{0} S+E^{\prime}$, where $E^{\prime}$ is an effective divisor which does not contain $S$ in its support and $n_{0}$ is a positive integer. We may assume that $E^{\prime}$ is Cartier, 
after possibly replacing $E$ by a larger divisor. For any sufficiently divisible positive integer $e$, we consider the following diagram:

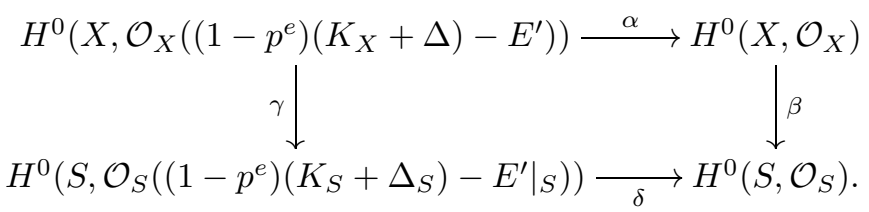

The fact that the diagram commutes follows from the fact that the different $\Delta_{S}$ coincides with the $F$-different 3 . Since $\left(S, \Delta_{S}\right)$ is globally $F$-regular, the map $\delta$ is surjective for $e \gg 0$. On the other hand $\gamma$ is sujrective since $-\left(K_{X}+\Delta\right)$ is $f$-ample and $e \gg 0$. Thus $\beta \circ \alpha$ is surjective. By Nakayama's lemma, so is $\alpha$ near $f(S)$. Since $\Delta \geq B$, we have

$$
H^{0}\left(X, \mathcal{O}_{X}\left(\left(1-p^{e}\right)\left(K_{X}+\Delta\right)-E^{\prime}\right)\right) \subseteq H^{0}\left(X, \mathcal{O}_{X}\left(\left(1-p^{e}\right)\left(K_{X}+B\right)-E\right)\right) .
$$

Thus, the surjectivity of

$$
H^{0}\left(X, \mathcal{O}_{X}\left(\left(1-p^{e}\right)\left(K_{X}+B\right)-E\right)\right) \rightarrow H^{0}\left(X, \mathcal{O}_{X}\right)
$$

for $e \gg 0$ follows.

Proposition 2.12 (4, Lemma 2.12]). Let $f: X \rightarrow T$ be a proper birational morphism of normal varieties such that $(X, \Delta)$ is globally $F$-regular over $T$.

Then $\left(T, \Delta_{T}=f_{*} \Delta\right)$ is strongly $F$-regular.

Proposition 2.13. Let $(X, \Delta)$ be a two dimensional Kawamata log terminal pair and let $q \in X$ be a closed point.

Then there exists a birational morphism $f: Y \rightarrow X$ such that

(1) $f$ is an isomorphism over $X \backslash\{q\}$,

(2) $E=f^{-1}(q)$ is irreducible,

(3) $-\left(K_{Y}+\Delta_{Y}+E\right)$ is f-ample, where $\Delta_{Y}=f_{*}^{-1} \Delta$,

(4) $\left(Y, \Delta_{Y}+E\right)$ is purely log terminal.

We follow closely the proof in [21, Remark 1]. The exceptional divisor $E$ is called the Kollár component of $(X, \Delta)$.

Proof. Without loss of generality we may assume that $X$ is affine. We first show that there exists an effective $\mathbb{Q}$-Cartier divisor $H$ on $X$ such that there exists exactly one exceptional divisor $E$ over $X$ with $f(E)=q$, such that $a(E, X, \Delta+H)=0$. Let $L \geq 0$ be a $\mathbb{Q}$-divisor on $X$ such that $(X, \Delta+L)$ is $\log$ canonical but not Kawamata $\log$ terminal at the point $q$. We may assume that $\lfloor\Delta+L\rfloor=0$. Let $g: Z \rightarrow X$ be a $\log$ resolution of $(X, \Delta+L)$. After possibly replacing $X$ by a smaller open subset, we may assume that $g$ is an isomorphism except over $q$. Then for any $t \in[0,1]$, we may write

$$
K_{Z}+\Delta_{Z}+t L_{Z}+\sum_{i=1}^{k} a_{i}(t) E_{i}=g^{*}\left(K_{X}+\Delta+t L\right),
$$

where $\Delta_{Z}=g_{*}^{-1} \Delta, L_{Z}=g_{*}^{-1} L$, and $E_{1}, \ldots, E_{k}$ are prime exceptional divisors. Furthermore, for each $i=1, \ldots, k$, the function $a_{i}(t)$ is linear, $a_{i}(t) \leq 1$ for any $t \in[0,1]$ and there exists $i \in\{1, \ldots, k\}$ such that $a_{i}(1)=1$. Let $b_{1}, \ldots, b_{k}$ be 
positive integers so that the divisor $A=-\sum_{i=1}^{k} b_{i} E_{i}$ is $g$-ample. Fix $\varepsilon>0$ to be a (small) rational number and choose $\delta>0$ to be sufficiently small so that

$$
A^{\prime}=\varepsilon g^{*} L+\delta A
$$

is effective. Let $c_{i}(t)=a_{i}(t)+\delta b_{i}$, for any $i=1, \ldots, k$. Then

$$
K_{Z}+\Delta_{Z}+t L_{Z}+A^{\prime}+\sum_{i=1}^{k} c_{i}(t) E_{i}=g^{*}\left(K_{X}+\Delta+(t+\varepsilon) L\right) .
$$

Then there exists $t_{0} \in(0,1)$ such that $\max \left\{c_{i}\left(t_{0}\right) \mid i=1, \ldots, k\right\}=1$. We may assume that $c_{1}\left(t_{0}\right)=1$. For any $i=2, \ldots, k$, let $\eta_{i} \geq 0$ be rational numbers such that $\eta_{i}>0$ if and only if $c_{i}\left(t_{0}\right)=1$. We may assume that $\eta_{i}$ (and $\varepsilon$ ) are sufficiently small so that the following holds: if we denote $c_{i}=c_{i}\left(t_{0}\right)-\eta_{i}, I=\{i=2, \ldots, k \mid$ $\left.c_{i}>0\right\}$, there exists an effective $\mathbb{Q}$-divisor

$$
A^{\prime \prime} \sim_{\mathbb{Q}} A^{\prime}+\sum_{i \geq 2} \eta_{i} E_{i}
$$

such that if

$$
\Theta=t_{0} L_{Z}+A^{\prime \prime}+\sum_{i \in I} c_{i} E_{i}
$$

then $\left(Z, E_{1}+\Delta_{Z}+\Theta\right)$ is purely log terminal.

We have

$$
K_{Z}+E_{1}+\Delta_{Z}+\Theta+\sum_{c_{i}<0} c_{i} E_{i} \sim_{\mathbb{Q}} g^{*}\left(K_{X}+\Delta+\left(t_{0}+\varepsilon\right) L\right) .
$$

Thus, if $H=g_{*} \Theta$, then $H \sim_{\mathbb{Q}}\left(t_{0}+\varepsilon\right) L,(X, \Delta+H)$ is $\log$ canonical and there exists exactly one exceptional divisor $E=E_{1}$ over $X$ such that $a(E, X, \Delta+H)=0$, as claimed.

Thus, there exists a birational morphism $f: Y \rightarrow X$ from a normal surface $Y$ which extracts $E$ (e.g. see [14, Proposition 3.1.2]). Note that the proof depends on the existence of a minimal model for a two dimensional dlt pair over $X$, which holds also in positive characteristic (e.g. see [20, Theorem 0.4]). In particular, $f$ admits a unique $f$-exceptional divisor $E$ and (1) and (2) follow. If $\Delta_{Y}$ and $H_{Y}$ are the strict transform of $\Delta$ and $H$ on $Y$ respectively, then $\left(Y, \Delta_{Y}+H_{Y}+E\right)$ is purely $\log$ terminal. Thus, (4) follows. We may write $f^{*} H=H_{Y}+m E$, for some $m>0$. Since

$$
K_{Y}+\Delta_{Y}+H_{Y}+E=f^{*}\left(K_{X}+\Delta+H\right) \sim_{\mathbb{Q}, f} 0,
$$

it follows that $-\left(K_{Y}+\Delta_{Y}+E\right) \sim_{\mathbb{Q}, f}-m E$. Since $-E$ is $f$-ample, (3) follows.

2.3. Complements. Similarly to [4], we will use Shokurov's theory of complements. The results in this section hold over any algebraically closed field.

Definition 2.14. Let $f: X \rightarrow Y$ be a proper morphism of normal varieties and let $(X, D=S+B)$ be a $\log$ canonical pair where $S=\lfloor D\rfloor$ and $B \geq 0$ is a $\mathbb{Q}$-divisor whose support does not contain any component of $S$. Let $n$ be a positive integer. We say that $D^{\prime}$ is an $n$-complement of $(X, D)$ over $Y$ if

(1) $n\left(K_{X}+D^{\prime}\right) \sim_{f} 0$,

(2) $\left(X, D^{\prime}\right)$ is $\log$ canonical, and

(3) $n D^{\prime} \geq n S+\lfloor(n+1) B\rfloor$.

If $D^{\prime} \geq D$, then the complement $D^{\prime}$ is called effective. If $(X, D)$ admits an $n$ complement over $Y$, then we say that $(X, D)$ is $n$-complementary over $Y$. 
Lemma 2.15. Let $n$ be a positive integer and let $I \subseteq(0,1]$ be a finite set. Let $X=\mathbb{P}^{1}$ and let $B$ be a $\mathbb{Q}$-divisor on $X$ such that

(1) the coefficients of $B$ belong to $D(I)$ and

(2) $-\left(K_{X}+B\right)$ is nef.

Then, there exists a positive integer $M$, depending only on $I$ and $n$, such that $(X, B)$ admits an nq-complement, for some positive integer $q \leq M$.

Proof. The result follows from [13, Proposition 5.7]. Note that although the result in 13 is stated only over the complex field, the proof is characteristic free.

Theorem 2.16. Let $I \subseteq[0,1] \cap \mathbb{Q}$ be a finite set of rational numbers. Then there exists a positive integer $N$ such that if $f: S \longrightarrow T$ is a proper map of normal surfaces and $(S, B)$ is a log pair such that

(1) $(S, B)$ is Kawamata log terminal,

(2) $-\left(K_{S}+B\right)$ is $f$-nef, and

(3) the coefficients of $B$ belong to $I$,

then there exists an effective $m$-complement $B^{c}$ of $(S, B)$ over $T$ such that $\left(S, B^{c}\right)$ is log canonical but not Kawamata log terminal and $m \leq N$.

Proof. Assume that $n$ is a positive integer such that $n a$ is integral for any $a \in I$. We want to show that there exists a positive integer $M$, depending only on $I$, such that if $(S, B)$ is a log pair which satisfies (1), (2) and (3), then $(S, B)$ is $n q$-complementary, for some positive integer $q \leq M$. Since $n q B$ is integral, any $n q$-complement of $(S, B)$ is automatically effective.

As in the proof of Proposition 2.13, there exists a nef $\mathbb{Q}$-divisor $H \geq 0$ such that $(S, B+H)$ admits exactly one exceptional divisor $E$ over $S$ with log discrepancy zero. We may assume that $(S, B+H)$ has no codimension one log canonical centers and Supp $H$ dose not contain any $f$-exceptional divisors. We denote $B^{\prime}=B+H$. Then $\left(S, B^{\prime}\right)$ is $\log$ canonical but not Kawamata log terminal. Let $g: X \rightarrow S$ be the birational morphism which extracts $E$ and let $h: X \rightarrow T$ be the induced morphism. We may write

$$
K_{X}+B_{X}^{\prime}=g^{*}\left(K_{S}+B^{\prime}\right) \quad \text { and } \quad K_{X}+B_{X}=g^{*}\left(K_{S}+B\right)
$$

for some $B_{X}^{\prime} \geq 0$ such that $B_{X}^{\prime}=E+\left\{B_{X}^{\prime}\right\}$, where $E=\left\lfloor B_{X}^{\prime}\right\rfloor$, and $B_{X} \leq B_{X}^{\prime}$. Note that $B_{X}$ is not necessarily effective. We denote by $H^{\prime}$ the strict transform of $H$ in $X$.

We denote by $B_{X}^{\prime \prime}=E \vee B_{X}$ the divisor obtained by replacing the coefficient corresponding to $E$ in $B_{X}$ by 1 and by keeping all the other coefficients of $B_{X}$ unchanged. Thus, we have

$$
B_{X} \leq B_{X}^{\prime \prime} \leq B_{X}^{\prime} \quad \text { and } \quad g_{*} B_{X}^{\prime \prime}=B
$$

since $B_{X}^{\prime}-B_{X}^{\prime \prime}=H^{\prime}$. Note that the difference of $B_{X}^{\prime \prime}$ and $B_{X}$ is only the coefficient of $E$. In addition, we have that $\left(X, B_{X}^{\prime \prime}\right)$ is plt.

We claim that $-\left(K_{X}+B_{X}^{\prime \prime}\right)$ is nef over $T$. Indeed if we have an $h$-exceptional curve $C$ with $-\left(K_{X}+B_{X}^{\prime \prime}\right) \cdot C<0$, then $C$ is contained in the support of $B_{X}^{\prime}-B_{X}^{\prime \prime}$ since $-\left(K_{X}+B_{X}^{\prime}\right)$ is nef over $T$. However, the support of $B_{X}^{\prime}-B_{X}^{\prime \prime}$ is equal to the support of $H^{\prime}$ which is not contracted by $h$. This is a contradiction, and therefore $-\left(K_{X}+B_{X}^{\prime \prime}\right)$ is nef over $T$.

Let $\Theta \geq 0$ be the $\mathbb{Q}$-divisor on $E$ defined by

$$
K_{E}+\Theta=\left.\left(K_{X}+B_{X}^{\prime \prime}\right)\right|_{E} .
$$


By Lemma 2.3, the coefficients of $\Theta$ belong to $D(I)$. Thus, by Lemma 2.15, there exists a positive integer $M$, depending only on $I$, such that $(E, \Theta)$ admits an $n q$ complement, for some positive integer $q \leq M$. Using the same methods as in [14, Proposition 6.0.6], it follows that $\left(X, B_{X}^{\prime \prime}\right)$ is $n q$-complementary over $T$. Note that the proof in [14] relies on the Kawamata-Viehweg vanishing theorem for proper birational morphisms of surfaces, which also holds in positive characteristic (e.g. see [4, Lemma 2.23]).

Thus, by [14, Proposition 4.3.1], $(S, B)$ is $n q$-complementary over $T$. Since $\left(X, B_{X}^{\prime \prime}\right)$ is not Kawamata log terminal, it follows that the complement that we obtained is log canonical but not Kawamata log terminal.

\section{UNIFORM BOUNDS ON THE $F$-PURE THRESHOLD FOR LINE ARRANGEMENTS IN $\mathbb{A}^{2}$}

Throughout the section we fix $X=\mathbb{A}_{k}^{2}=\operatorname{Spec} k[x, y]$ with maximal ideal at the origin $\mathfrak{m}=\langle x, y\rangle$.

For each $i=1, \ldots, \ell$, define a distinct divisor (line) $D_{i}=\operatorname{div}\left(x-\lambda_{i} y\right)$ going through the origin and choose integers $a_{i}>0$. Consider the divisor $D=\sum a_{i} D_{i}$. We fix $d=\sum a_{i}$.

Our first goal is to recall known bounds on the $F$-pure threshold of $(X, D)$. First we handle the highly pathological case when one of the $a_{i}$ s is very large.

Lemma 3.1. Suppose that for some $i, 2 a_{i} \geq d$.

Then

$$
\operatorname{fpt}(X, D)=\operatorname{lct}(X, D)=\frac{1}{a_{i}} .
$$

Proof. Note that this condition can be satisfied for at most two values of $i$. In the case where $2 a_{i}, 2 a_{j} \geq d, i \neq j$, then $a_{i}=a_{j}=d / 2$ and we have a simple normal crossings pair and the statement is obvious.

Thus we can assume that there is a unique $i$ with $2 a_{i} \geq d$. Set $D^{\prime}=\sum_{j \neq i} a_{j} D_{j}$ and fix $d^{\prime}=\sum_{j \neq i} a_{j}$. By $F$-adjunction [7, 15], it is sufficient to verify that $\left(D_{i},\left.\frac{1}{a_{i}} D^{\prime}\right|_{D_{i}}\right)$ is sharply $F$-pure. But $D_{i} \cong \mathbb{A}^{1}$ and $\left.D^{\prime}\right|_{D_{i}}=d^{\prime} O$, where $O$ is the origin. But $d^{\prime} / a_{i} \leq 1$, which proves that $\left(D_{i},\left.\frac{1}{a_{i}} D^{\prime}\right|_{D_{i}}\right)$ is sharply $F$-pure and completes the proof of the lemma.

We recall the following result independently obtained by Hara [6] and Monsky [12. Similar bounds (which are good enough for our purposes) can also be obtained by modifying the method of [1, Lemmas 3.3, 3.4], which also works in higher dimensions.

Theorem 3.2 ([6, Proposition 3.3], [12, Theorem 17]). Suppose $D=\sum_{i=1}^{\ell} a_{i} D_{i}$ is a line arrangement through the origin in $X=\mathbb{A}^{2}$ as above with $d=\sum a_{i}$. If $2 a_{i}<d$ for all $i$, then

$$
\operatorname{fpt}(X, D) \geq \frac{2 p-\ell+2}{d p} .
$$

As an easy corollary we obtain the following.

Corollary 3.3. Fix $X=\mathbb{A}^{2}$. Suppose that $\Lambda$ is a set of rational numbers in $(0,1]$ and $t_{0}$ is a positive real constant such that for each integer $d>2$, if $\lambda<\frac{2}{d}$ for some 
$\lambda \in \Lambda$, then $\frac{2}{d}-\lambda \geq t_{0}$. Suppose

$$
p \geq \frac{1}{t_{0}}
$$

Then for any line arrangement $\left(X, D:=\sum_{i=1}^{\ell} a_{i} D_{i}\right)$ with integer $a_{i}$ so that for some $\lambda \in \Lambda$ we have $(X, \lambda D)$ is Kawamata log terminal, then $(X, \lambda D)$ is strongly F-regular.

Before proving this corollary we make two observations. Firstly, we notice that $\Lambda$ is bounded away from zero by hypothesis (since $\frac{2}{d}$ converges to zero). Secondly, the condition on $\Lambda$ is satisfied for any ACC subset of $(0,1]$ which is also bounded away from zero.

Proof. Note that the cases of $\ell=1,2$ are uninteresting as then $(X, D)$ is SNC, and so $(X, \lambda D)$ is Kawamata log terminal if and only if it is strongly $F$-regular. We fix $D=\sum_{i=1}^{\ell} a_{i} D_{i}$ with $d=\sum_{i=1}^{\ell} a_{i}$ and fix $\lambda \in \Lambda$ such that $(X, \lambda D)$ is Kawamata $\log$ terminal. We need to show that $\lambda<\operatorname{fpt}(X, D)$; we know that $\lambda<$ $\operatorname{lct}(X, D)$. Note that we may assume that $2 a_{i}<d=\sum_{i=1}^{\ell} a_{i}$ for all $i$ since otherwise $\operatorname{lct}(X, D)=\operatorname{fpt}(X, D)=\frac{1}{a_{i}}$ by Lemma 3.1. Thus we see that $\operatorname{lct}(X, D)=\frac{2}{d}$ so that $\frac{2}{d}-\lambda \geq t_{0} \geq \frac{1}{p}$.

Now observe that

$$
\lambda \leq \frac{2}{d}-\frac{1}{p} \leq \frac{2}{d}-\frac{\ell}{d p}=\frac{2 p-\ell}{d p}<\frac{2 p-\ell+2}{d p} \leq \operatorname{fpt}(X, D),
$$

where the last inequality comes from Theorem 3.2 . This completes the proof.

Example 3.4. We cannot weaken the hypothesis of Corollary 3.3 to simply that $\Lambda$ is an ACC set. For example, suppose that $\Lambda=\left\{\frac{1}{n}\right\}_{n \in \mathbb{N}}$. Then for each prime integer $p$, set $D$ to be the set of $p+1$ distinct lines of $\mathbb{A}_{\mathbb{F}_{p}}^{2}$. In other words, $D=$ $\operatorname{div}(x y(x+y)(x+2 y) \ldots(x+(p-1) y))$. Then it is easy to check that $\operatorname{fpt}(X, D)=$ $\frac{1}{p}<\frac{2}{p+1}=\operatorname{lct}(X, D)$. In particular, for each prime $p,\left(X, \frac{1}{p} D\right)$ is not strongly $F$-regular even though it is Kawamata log terminal.

Example 3.5 (Standard coefficients). Suppose that

$$
\Lambda=D(\emptyset)=\left\{\frac{n-1}{n} \mid n \in \mathbb{N}\right\}
$$

is the set of standard coefficients. Then the only element $\lambda \in \Lambda$ with $\lambda<2 / d$, for some $d>2$, is $\lambda=\frac{2-1}{2}=1 / 2$, for $d=3$. In particular, we see that

$$
1 / 6=2 / 3-1 / 2=\min \{2 / d-\lambda>0 \mid d>2, \lambda \in \Lambda\} .
$$

It follows from Corollary 3.3 that if $p>6=\frac{1}{1 / 6}$ and $\lambda \in \Lambda$, then $(X, \lambda D)$ is Kawamata log terminal if and only if it is strongly $F$-regular where $X=\mathbb{A}^{2}$ and $D$ is a line arrangement.

Example 3.6 (Simple hyperstandard coefficients). Fix a positive integer $n$. Suppose that

$$
\begin{aligned}
\Lambda_{n} & =D\left(\left\{\frac{1}{n}\right\}\right) \\
& =D\left(\left\{\frac{a}{n} \mid a=0, \ldots, n\right\}\right) \\
& =\left\{\frac{n m+a-n}{n m} \mid m \in \mathbb{N}, a=0, \ldots, n\right\} \\
& =\left\{0, \frac{1}{n}, \frac{2}{n}, \ldots, \frac{n-1}{n}, 1, \frac{1}{2}, \frac{n+1}{2 n}, \ldots, \frac{2 n-1}{2 n}, \frac{2}{3}, \frac{2 n+1}{3 n}, \ldots, \frac{3 n-1}{3 n}, \frac{3}{4}, \ldots\right\} .
\end{aligned}
$$


We want to find the minimum of $\frac{2}{d}-\lambda$, with $\lambda \in \Lambda_{n}$ and $d \in \mathbb{N}$ such that $d>2$ and $\frac{2}{d-\lambda}>0$. Note that there are only finitely many $\lambda \in \Lambda_{n}$ which are less than $2 / d$ (and all of those are before the $\frac{2}{3}$ in the list above), and we only must consider $d=3, \ldots, 2 n-1$. Also note we may as well consider $n \geq 3$ since $n=1,2$ are already covered by Example 3.5 .

For $d=3$, we see that

$$
m_{3}=\min \left\{\frac{2}{3}-\lambda>0 \mid \lambda \in \Lambda_{n}\right\}=\min \left\{\frac{2}{3}-\frac{n+a}{2 n}>0 \mid a=0, \ldots, n\right\} \geq \frac{1}{6 n} .
$$

Likewise for each $2 n-1 \geq d>3$ we see that

$$
m_{d}=\min \left\{\frac{2}{d}-\lambda>0 \mid \lambda \in \Lambda_{n}\right\}=\min \left\{\frac{2}{d}-\frac{a}{n}>0 \mid a=0, \ldots, n\right\} \geq \frac{1}{d n} \geq \frac{1}{(2 n-1) n} .
$$

We set $m=\min \left\{m_{3}, m_{4}, \ldots, m_{2 n-1}\right\}$.

Notice that

$$
\frac{2}{2 n-1}-\frac{1}{n}=\frac{2 n-(2 n-1)}{(2 n-1) n}=\frac{1}{(2 n-1) n} .
$$

It follows easily that $m=\min \left(\frac{1}{(2 n-1) n}, m_{3}\right)$. If $n \geq 4$, then $2 n-1>6$ in which case $m=\frac{1}{(2 n-1) n}$. Finally, we consider $n=3$ explicitly. Note that for $m_{3}$ we are minimizing $\frac{2}{3}-\frac{3+a}{6}$, and we see that it is minimized when $a=0$, in which case $m_{3}=\frac{1}{6} \geq \frac{1}{(2 n-1) n}=\frac{1}{15}$.

Combining this example with Corollary 3.3 yields the following result.

Corollary 3.7. Suppose that $X=\mathbb{A}^{2}$ and $D$ is a line arrangement through the origin. If $n \geq 3, \Lambda=D\left(\left\{\frac{1}{n}\right\}\right)$ and $p>2 n^{2}-n$, then for any $\lambda \in \Lambda$, we have that $(X, \lambda D)$ is Kawamata log terminal if and only if it is strongly F-regular.

For our application, we need to handle the case when the coefficients of $D$ are rational numbers from $\Lambda$. This can be more complicated as we shall see.

Corollary 3.8. Fix $X=\mathbb{A}^{2}$. Let $\varepsilon>0$ be a rational number and let $\Gamma \subseteq(\varepsilon, 1) \cap \mathbb{Q}$ be a subset which satisfies $A C C$.

Then there exists a positive constant $p_{0}$ depending only on $\Gamma$ (and in particular on $\varepsilon)$ such that if the line arrangement $\left(X, B:=\sum_{i=1}^{\ell} q_{i} D_{i}\right)$ is a Kawamata log terminal pair defined over a field of characteristic $p>p_{0}$ and such that $q_{1}, \ldots, q_{\ell} \in$ $\Gamma$, then $(X, B)$ is strongly $F$-regular.

Proof. Suppose not. Then, for any $i \in \mathbb{N}$ there exists a pair

$$
\left(X, B_{i}=\sum_{j=1}^{\ell_{i}} q_{i, j} D_{i, j}\right)
$$

defined over an algebraically closed field of characteristic $p_{i}$, which is Kawamata $\log$ terminal but not strongly $F$-regular and such that $\lim p_{i}=\infty$ and $q_{i, j} \in \Gamma$.

Since $\left(X, B_{i}\right)$ is Kawamata log terminal and $q_{i, j}>\varepsilon$, it follows that

$$
\ell_{i}<\frac{2}{\varepsilon}
$$

Thus, after possibly taking a subsequence, we may assume that $\ell_{i}=\ell>2$ is constant. By Corollary 3.3. we may assume that the set of coefficients

$$
\left\{q_{i, j} \mid i \in \mathbb{N}, \quad j=1, \ldots, \ell\right\}
$$


is not finite, and since $\Gamma$ satisfies ACC, after possibly taking a subsequence, we may assume that $q_{i}:=\sum_{j=1}^{\ell} q_{i, j}$ is a strictly decreasing sequence. Note we may also assume that $q_{i}>2 q_{i, j}$ for all $i, j$. We may write

$$
B_{i}=\frac{1}{c_{i}} \sum_{j=1}^{\ell} b_{i, j} D_{i, j}
$$

for some positive integers $c_{i}, b_{i, 1}, \ldots, b_{i, \ell}$. Let $\lambda_{i}=\frac{1}{c_{i}}$ and let $G_{i}=\sum_{j=1}^{\ell} b_{i, j} D_{i, j}$ so that $B_{i}=\lambda_{i} G_{i}$. Let $d_{i}=\sum_{j=1}^{\ell} b_{i, j}=c_{i} q_{i}$. Then by assumption, we have that for any $i \in \mathbb{N}$,

$$
\operatorname{fpt}\left(X, G_{i}\right) \leq \lambda_{i}<\operatorname{lct}\left(X, G_{i}\right) .
$$

Thus, by Lemma 3.1 and Theorem 3.2 , we have

$$
\frac{2 p_{i}-\ell+2}{d_{i} p_{i}} \leq \lambda_{i}<\frac{2}{d_{i}} \text {. }
$$

In particular,

$$
1-\frac{\ell-2}{2 p_{i}}=\frac{2 p_{i}-\ell+2}{2 p_{i}} \leq \frac{d_{i} \lambda_{i}}{2}=\frac{d_{i}}{2 c_{i}}=\frac{q_{i}}{2}<1 .
$$

Since $\lim p_{i}=\infty$ and $q_{i}$ is a strictly decreasing sequence, we get a contradiction.

For our purposes however, we need to handle sets of the form $D(I)$ because those sorts of sets will occur via adjunction.

Corollary 3.9. Let $I \subseteq(0,1) \cap \mathbb{Q}$ be a finite subset and let $\Gamma=D(I)$. Fix $\varepsilon=\min I \cup\left\{\frac{1}{2}\right\}$ and

$$
\mathcal{S}=\left\{q=\sum_{i=1}^{\ell} q_{i} \mid q_{i} \in \Gamma \text { such that } \sum_{i \neq j} q_{i}>1 \text { for any } j=1, \ldots, \ell\right\} \cap(0,2) .
$$

Define $Q=\max \mathcal{S}$ and $p_{0}=\left\lfloor\frac{1-\varepsilon}{\varepsilon} \cdot \frac{1}{1-Q / 2}\right\rfloor$ and observe it depends only on $I$.

If the line arrangement $\left(X, B:=\sum_{i=1}^{\ell} q_{i} D_{i}\right)$ is a Kawamata log terminal pair defined over a field of characteristic $p>p_{0}$ and such that $q_{1}, \ldots, q_{\ell} \in \Gamma$, then $(X, B)$ is strongly F-regular.

Note that although $\mathcal{S}$ is not finite, it is easy to check that since $I$ is finite, the maximum of $\mathcal{S}$ is attained and in particular $Q<2$.

Proof. Assume that $\left(X, B=\sum_{i=1}^{\ell} q_{i} D_{i}\right)$ is a Kawamata log terminal pair which is not strongly $F$-regular and such that $q_{i} \in \Gamma$.

Assume first that there exists $j \in\{1, \ldots, \ell\}$ such that $\sum_{i \neq j} q_{i} \leq 1$. Let

$$
C=D_{j}+\sum_{i \neq j} q_{i} D_{i}
$$

Then $C \geq B$ and $\left(D_{j},\left.\left(C-D_{j}\right)\right|_{D_{j}}\right)=\left(\mathbb{A}^{1}, \sum_{i \neq j} q_{i} O\right)$, where $O \in \mathbb{A}^{1}$ is the origin. In particular, $\left(\mathbb{A}^{1}, \sum_{i \neq j} q_{i} O\right)$ is sharply $F$-pure and by $F$-adjunction it follows that $(X, C)$ is also sharply $F$-pure. By Lemma 2.10 , it follows that $(X, B)$ is strongly $F$-regular, a contradiction.

We now assume that $\sum_{i \neq j} q_{i}>1$ for any $j \in\{1, \ldots, \ell\}$. Let $q=\sum_{j=1}^{\ell} q_{i}$. Since $(X, B)$ is Kawamata $\log$ terminal, we have that $q<2$ and $\ell<\frac{2}{\varepsilon}$. In particular, $q \in \mathcal{S}$ and therefore $q \leq Q$. 
We may write

$$
B=\frac{1}{c} \sum_{i=1}^{\ell} b_{i} D_{i}
$$

for some positive integers $c, b_{1}, \ldots, b_{\ell}$. Let $\lambda=\frac{1}{c}$ and let $G=\sum_{j=1}^{\ell} b_{i} D_{i}$ so that $B=\lambda G$. Let $d=\sum_{j=1}^{\ell} b_{i}=c q$.

Thus, by Lemma 3.1 and Theorem 3.2 we have

$$
\frac{2 p-\ell+2}{d p} \leq \lambda<\frac{2}{d}
$$

In particular,

$$
1-\frac{\ell-2}{2 p}=\frac{2 p-\ell+2}{2 p} \leq \frac{d}{2 c}=\frac{q}{2}<1
$$

and it follows that

$$
p \leq \frac{1}{2}\left(\frac{\ell-2}{1-q / 2}\right)<\frac{\frac{2}{\varepsilon}-2}{2-Q}
$$

Thus, the claim follows.

Remark 3.10. Note that while it might be natural to require that $I$ is an arbitrary DCC set (since if $I$ is finite, $D(I)$ is DCC), there are difficulties with this assumption. For instance if $I$ is a DCC set containing $\left\{\frac{2}{3}-\frac{1}{n}\right\}$, then it is not difficult to see that the conclusion of Corollary 3.8 fails to hold for a configuration of three lines (since if $p \equiv 2 \bmod 3$, then fpt $<$ lct $=\frac{2}{3}$ ).

The bound of Corollary 3.9 is far from sharp, as the following example shows. Thus we hope that the bound can be substantially improved.

Example 3.11. Suppose that $I=\emptyset$. Then it is not difficult to see that $\varepsilon=\frac{1}{2}$ and $Q=\max \mathcal{S}=\frac{1}{2}+\frac{2}{3}+\frac{4}{5}=59 / 30$. Hence we can take $p_{0}=30$. This is far from optimal; see [4, Section 3].

Example 3.12. Next suppose that $I=\left\{\frac{1}{3}\right\}$. We see that $\varepsilon=\frac{1}{3}$. We consider three values for $\ell=3,4,5$ (notice $\ell<\frac{2}{1 / 3}=6$ ). Note that the list of valid $q_{i}$ as in Example 3.6 is

$$
\begin{gathered}
\left\{1, \frac{1}{2}, \frac{1}{3}, \frac{2}{3}, \frac{4}{6}, \frac{5}{6}, \frac{6}{9}, \frac{7}{9}, \frac{8}{9}, \frac{3}{4}, \frac{10}{12}, \frac{11}{12}, \frac{4}{5}, \frac{13}{15}, \frac{14}{15}, \frac{5}{6}, \frac{16}{18}, \frac{17}{18}, \frac{6}{7}, \frac{19}{21}, \frac{20}{21}, \frac{7}{8}, \frac{22}{24}, \frac{23}{24}, \frac{8}{9}, \ldots\right\} \\
=\left\{1, \frac{1}{2}, \frac{1}{3}, \frac{2}{3}, \frac{5}{6}, \frac{7}{9}, \frac{8}{9}, \frac{3}{4}, \frac{11}{12}, \frac{4}{5}, \frac{13}{15}, \frac{14}{15}, \frac{17}{18}, \frac{6}{7}, \frac{19}{21}, \frac{20}{21}, \frac{7}{8}, \frac{23}{24}, \frac{25}{27}, \frac{26}{27}, \ldots\right\} .
\end{gathered}
$$

First we consider $\ell=5$. Then we see immediately that any $q_{i}<\frac{2}{3}$ (since the minimum value of the other $q_{j}$ is $\frac{1}{3}$ ). But the only such elements of $D(I)$ are $\left\{\frac{1}{3}, \frac{1}{2}\right\}$ and the $Q$ associated to any such sum is even smaller than the $I=\emptyset$ case.

Next we consider $\ell=4$. Note we cannot have three of the $q_{i}$ equalling $\frac{1}{3}$ since then their sum would be $\leq 1$. Hence at most two of the $q_{i}$ are $\frac{1}{3}$, and so since the smallest value a third $q_{i}$ can be is $\frac{1}{2}$, we see that any such $q_{i}<2-\left(\frac{1}{3}+\frac{1}{3}+\frac{1}{2}\right)=\frac{5}{6}$. The only values of $D(I)$ less than $\frac{5}{6}$ are $\left\{\frac{1}{3}, \frac{1}{2}, \frac{2}{3}, \frac{7}{9}, \frac{3}{4}, \frac{4}{5}\right\}$. A quick computation shows that $Q=\frac{1}{3}+\frac{1}{3}+\frac{1}{2}+\frac{4}{5}=\frac{59}{30}$ again, and we recover nothing new compared to the $I=\emptyset$ case.

Finally we consider $\ell=3$. Suppose now that $q_{1} \leq q_{2} \leq q_{3}$. Of course, at least one of the $q_{i}<\frac{2}{3}$ since their sum is less than 2 , and so either $q_{1}=\frac{1}{3}$ or $q_{1}=\frac{1}{2}$. 
Suppose first that $q_{1}=\frac{1}{3}$. It follows that $q_{2}<\frac{5}{6}$, and also since $q_{1}+q_{2}>1$ we know that $q_{2} \in\left\{\frac{7}{9}, \frac{3}{4}, \frac{4}{5}\right\}$. We break this up into cases.

$q_{1}=\frac{1}{3}, q_{2}=\frac{7}{9}$ : and so $q_{1}+q_{2}=\frac{10}{9}$ and $q_{3}<\frac{8}{9}$. The largest possible $p_{3}$ is then $\frac{13}{15}$. We see that $Q \geq \frac{1}{3}+\frac{7}{9}+\frac{13}{15}=\frac{89}{45}$.

$q_{1}=\frac{1}{3}, q_{2}=\frac{3}{4}$ : and so $q_{1}+q_{2}=\frac{13}{12}$ and $q_{3}<\frac{11}{12}$. The largest possible $q_{3}$ is then $\frac{19}{21}$. We see that $Q \geq \frac{1}{3}+\frac{3}{4}+\frac{19}{21}=\frac{167}{84}$.

$q_{1}=\frac{1}{3}, q_{2}=\frac{4}{5}$ : and so $q_{1}+q_{2}=\frac{17}{15}$ and $q_{3}<\frac{13}{15}$. The largest possible $q_{3}$ is then $\frac{6}{7}$. We see that $Q \geq \frac{1}{3}+\frac{4}{5}+\frac{6}{7}=\frac{209}{105}$.

Now we handle the case that $q_{1}=\frac{1}{2}$. It follows that $q_{2}<\frac{3}{4}$ and also since $q_{1}+q_{2}>1$, we know that $q_{2} \in\left\{\frac{2}{3}\right\}$. Hence we see that $q_{1}+q_{2}=\frac{7}{6}$, and so $q_{3}<\frac{5}{6}$. The largest possible $q_{3}$ is then $\frac{4}{5}$, and we see that $Q \geq \frac{1}{2}+\frac{2}{3}+\frac{4}{5}=\frac{29}{30}$.

Summarizing, we see that $Q=\frac{209}{105}$. Since $\varepsilon=\frac{1}{3}$ we see that

$$
p_{0}=\left(\frac{1-(1 / 3)}{1 / 3}\right)\left(\frac{1}{1-209 / 210}\right)=2 \cdot 210=420 .
$$

This completes the example.

Removing the explicit bound of Theorem 1.1] we can restate Corollary [3.9] as:

Corollary 3.13. Let $I \subseteq(0,1) \cap \mathbb{Q}$ be a finite subset and let $\Gamma=D(I)$.

Then there exists a positive constant $p_{0}$ depending only on $\Gamma$ such that if the line arrangement $\left(X:=\mathbb{A}^{2}, B:=\sum_{i=1}^{\ell} q_{i} D_{i}\right)$ is a Kawamata log terminal pair defined over a field of characteristic $p>p_{0}$ and $q_{1}, \ldots, q_{\ell} \in \Gamma$, then $(X, B)$ is strongly F-regular.

\section{Global to local}

The aim of this section is to prove Theorem 1.1. We begin with the following:

Corollary 4.1. Let $I \subseteq(0,1) \cap \mathbb{Q}$ be a finite subset and let $\Gamma=D(I)$.

Then there exists a positive constant $p_{0}$ depending only on $I$ such that if $\left(\mathbb{P}^{1}, B:=\right.$ $\left.\sum_{i=1}^{\ell} q_{i} P_{i}\right)$ is a log Fano pair defined over an algebraically closed field of characteristic $p>p_{0}$ and such that the coefficients of $B$ belong to $\Gamma$, then $\left(\mathbb{P}^{1}, B\right)$ is globally F-regular.

Proof. By considering the cone associated to $\mathcal{L}=\mathcal{O}_{\mathbb{P}^{1}}(1)$, Proposition 2.9 implies that $\left(\mathbb{P}^{1}, \sum_{i=1}^{\ell} q_{i} P_{i}\right)$ is globally $F$-regular (resp. $\log$ Fano) if and only if $\left(\mathbb{A}^{2}, \sum_{i=1}^{\ell} q_{i} D_{i}\right)$ is strongly $F$-regular (resp. Kawamata log terminal), where $D_{1}, \ldots$, $D_{\ell}$ are some suitable distinct lines through the origin.

Thus, Corollary 3.13 implies the claim. In fact we can even choose $p_{0}$ effectively using the bound of Corollary 3.9 .

We can now proceed with the proof of Theorem 1.1 .

Proof of Theorem 1.1 . Let $B$ be a $\mathbb{Q}$-divisor such that $(X, B)$ is Kawamata log terminal. We may assume that $X$ is affine and let $q \in X$ be a closed point. By Proposition 2.13, we can take a birational morphism $f: Y \rightarrow X$ such that

(1) $f$ is isomorphic over $X \backslash\{q\}$,

(2) $E=f^{-1}(q)$ is irreducible,

(3) $-\left(K_{Y}+B_{Y}+E\right)$ is $f$-ample, where $B_{Y}=f_{*}^{-1} B$,

(4) $\left(Y, B_{Y}+E\right)$ is purely log terminal. 
In particular, if we write $K_{E}+B_{E}=\left.\left(K_{Y}+B_{Y}+E\right)\right|_{E}$, then $\left(E, B_{E}\right)$ is Kawamata $\log$ terminal, and by Lemma 2.3 and Lemma 2.2. the coefficients of $B_{E}$ belong to $D(I)$. Thus, Corollary 4.1 implies that there exists a positive integer $p_{0}$ such that $\left(E, B_{E}\right)$ is globally $F$-regular, if $p>p_{0}$. Then Proposition 2.11 implies that $\left(Y, B_{Y}\right)$ is globally $F$-regular over $X$. Thus, by Proposition 2.12 $(X, B)$ is strongly $F$-regular.

\section{ON THE EXISTENCE OF FLIPS}

This section is highly inspired by Section 3 in [4. In particular, our goal is to extend [4, Theorem 3.1] by the following:

Theorem 5.1. Let $I \subseteq(0,1)$ be a finite set. Then there exists a prime $p_{I}$, depending only on $I$, such that if $(S, B)$ is a pair over an algebraically closed field of characteristic $p>p_{I}$ and $f: S \longrightarrow T$ is a morphism of normal surfaces, such that

(1) $(S, B)$ is Kawamata log terminal,

(2) $-\left(K_{S}+B\right)$ is $f$-nef, and

(3) the coefficients of $B$ belong to $I$,

then $(S, B)$ is globally $F$-regular over $T$.

Following the same steps as in $44, \S 4.4]$, it is possible to show that Theorem 5.1 implies Theorem 1.2

We now begin the proof of Theorem [5.1. Let $B^{c} \geq B$ and $N$ be as in Theorem 2.16 and let $\nu: \tilde{S} \rightarrow S$ be a smooth dlt model of $\left(S, B^{c}\right)$. We may write

$$
K_{\tilde{S}}+B_{\tilde{S}}^{c}=\nu^{*}\left(K_{S}+B^{c}\right) \quad \text { and } \quad K_{\tilde{S}}+B_{\tilde{S}}=\nu^{*}\left(K_{S}+B\right),
$$

where $\left(S, B_{\tilde{S}}^{c}\right)$ is divisorially $\log$ terminal and $B_{\tilde{S}} \leq B_{\tilde{S}}^{c}$. Note that $B_{\tilde{S}}$ is not necessarily effective. By Theorem [2.16, it follows that $C=\left\lfloor B_{\tilde{S}}^{c}\right\rfloor$ is not zero. We first assume that $\left(\tilde{S}, B_{\tilde{S}}^{c}\right)$ is purely log terminal.

Claim 5.2. There exist a finite subset $J \subseteq[0,1]$ depending only on $I$ and a $\mathbb{Q}$-divisor $B_{\tilde{S}}^{*} \geq 0$ such that

(1) $B_{\tilde{S}} \leq B_{\tilde{S}}^{*} \leq B_{\tilde{S}}^{c}$

(2) the coefficients of $B_{\tilde{S}}^{*}$ belong to $J$,

(3) $\left\lfloor B_{\tilde{S}}^{*}\right\rfloor=C$, and

(4) $-\left(K_{\tilde{S}}+B_{\tilde{S}}^{*}\right)$ is nef over $T$ and $-\left.\left(K_{\tilde{S}}+B_{\tilde{S}}^{*}\right)\right|_{C}$ is ample.

We now prove how Claim 5.2 implies Theorem 5.1. We may write

$$
\left.\left(K_{\tilde{S}}+B_{\tilde{S}}^{*}\right)\right|_{C}=K_{C}+B_{C}^{*} .
$$

In particular $\left(C, B_{C}^{*}\right)$ is log Fano. By Corollary 4.1, there exists $p_{0}$ depending only on $J$ (and therefore depending only on $I$ ) such that if the characteristic of the base field is greater than $p_{0}$, then $\left(C, B_{C}^{*}\right)$ is globally $F$-regular. Thus, Theorem 5.1 follows immediately from Proposition 2.11.

We now proceed with the proof of Claim 5.2 The following lemma is obvious:

Lemma 5.3. Let $I \subseteq[0,1]$ be a finite set. Then for any $\varepsilon>0$, the set $D(I) \cap$ $(0,1-\varepsilon)$ is finite. 
In particular, because our $I$ is finite, we apply Lemma 5.3 for $\varepsilon=1 / N$. Then there exists a sufficiently small rational number $x>0$ such that for any $a \in D(I)$ we have that

$$
a \notin\left(\frac{p-x}{q-x}, \frac{p}{q}\right) \quad \text { for any } q=2, \ldots, N, \quad p=1, \ldots, q-1 .
$$

We use $J$ to denote

$$
\left\{\frac{p-x}{q-x} \mid q=2, \ldots, N, \quad p=1, \ldots, q-1\right\} \cup\left\{\frac{p}{q} \mid q=2, \ldots, N, \quad p=1, \ldots, q-1\right\} .
$$

Let $g=f \circ \nu: \tilde{S} \rightarrow T$. Note that $C$ is $g$-exceptional. In addition, since $(S, B)$ is Kawamata log terminal and $-\left(K_{S}+B\right)$ is $f$-nef, it follows that $\left(T, B_{T}\right)$ is Kawamata log terminal. Thus, since $g$ factors through the minimal resolution of $S$, it follows that the graph associated to the exceptional divisor of $g$ is a tree and its components are smooth rational curves which meet transversally with each other. We now proceed similarly as in [4, Lemma 3.3]. Let $D$ be the connected component of $B_{\tilde{S}}^{c}-B_{\tilde{S}}$ which contains $C$. Assume by contradiction that $D$ is $g$-exceptional. Then

$$
\begin{aligned}
D^{2} & =\left(B_{\tilde{S}}^{c}-B_{\tilde{S}}\right) \cdot D \\
& =\left(K_{\tilde{S}}+B_{\tilde{S}}^{c}\right) \cdot D-\left(K_{\tilde{S}}+B_{\tilde{S}}\right) \cdot D \\
& =-\left(K_{S}+B\right) \cdot \nu_{*} D \geq 0,
\end{aligned}
$$

which is a contradiction. Thus, there exists a curve contained in the support of $D$ which is not $g$-exceptional. Thus, there exists a chain of smooth rational curves in $\tilde{S}$

$$
C_{0}=C, C_{1}, \ldots, C_{k},
$$

such that $C_{i-1} \cdot C_{i}=1$ if $i=1, \ldots, k-1, C_{0}, \ldots, C_{k-1}$ are $g$-exceptional, $C_{k}$ is not $g$ exceptional and $C_{1}, \ldots, C_{k}$ are contained in the support of $B_{\tilde{S}}^{c}$. Let $q \in\{2, \ldots, N\}$ such that $q\left(K_{\tilde{S}}+B_{\tilde{S}}^{c}\right) \sim_{\mathbb{Z}, S} 0$. Then the coefficients of $B_{\tilde{S}}^{c}-C$ are of the form $p / q$ for some $p \in\{1, \ldots, q-1\}$. We define $B_{\tilde{S}}^{*}$ by replacing, for each $i=1, \ldots, k$, the coefficient in $B_{\tilde{S}}^{c}$ of the form $p / q$ by the coefficient $(p-x) /(q-x)$, and for each other curve in the support of $B_{\tilde{S}}^{c}$ we do not change the coefficient. In particular, we have that $B_{\tilde{S}}^{*} \leq B_{\tilde{S}}^{c},\left\lfloor B_{\tilde{S}}^{*}\right\rfloor=C$ and the coefficients of $B_{\tilde{S}}^{*}-C$ belong to $J$.

It follows immediately that $-\left(K_{\tilde{S}}+B_{\tilde{S}}^{*}\right) \cdot C<0$. Thus, $-\left.\left(K_{\tilde{S}}+B_{\tilde{S}}^{\prime}\right)\right|_{C}$ is ample.

We now show that $-\left(K_{\tilde{S}}+B_{\tilde{S}}^{*}\right)$ is nef over $T$. To this end, we use the same argument and the same notation as in [4, Lemma 3.4]. It is enough to show that $-\left(K_{\tilde{S}}+B_{\tilde{S}}^{*}\right) \cdot C_{j} \geq 0$ for any $j=1, \ldots, k-1$. For any $j<k-1$, it holds that

$$
\frac{p_{j-1}}{q}+\frac{p_{j+1}}{q}+\frac{p_{j}}{q} C_{j}^{2}+\frac{r}{q}-2-C_{j}^{2}=0
$$

from which

$$
\left(K_{\tilde{S}}+B_{\tilde{S}}^{c}\right) \cdot C_{j}=0
$$

This implies that

$$
p_{j-1}+p_{j+1}+r-2 q+\left(p_{j}-q\right) C_{j}^{2}=0,
$$

which, in turn, implies that

$$
\frac{p_{j-1}-x}{q-x}+\frac{p_{j+1}-x}{q-x}+\frac{p_{j}-x}{q-x} C_{j}^{2}+\frac{r}{q}-2-C_{j}^{2} \leq 0 .
$$


Thus, $-\left(K_{\tilde{S}}+B_{\tilde{S}}^{c}\right) \cdot C_{i} \geq 0$ for any $j=1, \ldots, k-2$. A similar calculation yields $-\left(K_{\tilde{S}}+B_{\tilde{S}}^{c}\right) \cdot C_{k-1}<0$ and the claim follows.

Finally, by the same proof as [4, Lemma 3.5] and since by assumption the coefficients of $B$ are not contained within the interval $\left(\frac{p-x}{q-x}, \frac{p}{q}\right)$ for any coefficient $p / q$ of $B_{\tilde{S}}^{c}$, it follows that $B_{\tilde{S}} \leq B_{\tilde{S}}^{*}$. If $\left(\tilde{S}, B_{\tilde{S}}^{c}\right)$ is purely log terminal, then $\left(\tilde{S}, B_{\tilde{S}}^{*}\right)$ is also purely log terminal, and therefore Claim 5.2 follows.

We now assume that $\left(\tilde{S}, B_{\tilde{S}}^{c}\right)$ is not purely log terminal. Let

$$
J=\left\{\frac{p}{q} \mid q=2, \ldots, N, \quad p=1, \ldots, q-1\right\} .
$$

Proceeding exactly as in [4, p. 19], we can find a $\mathbb{Q}$-divisor $B_{\tilde{S}}^{*} \geq 0$ which satisfies the following properties:

(1) $\left(S, B_{\tilde{S}}^{*}\right)$ is purely log terminal, and $C=\left\lfloor B_{\tilde{S}}^{*}\right\rfloor$ is a smooth rational curve,

(2) $B_{\tilde{S}} \leq B_{\tilde{S}}^{*} \leq B_{\tilde{S}}^{c}$,

(3) $-\left(K_{\tilde{S}}+B_{\tilde{S}}^{*}\right)$ is nef over $T$,

(4) $-\left.\left(K_{\tilde{S}}+B_{\tilde{S}}^{*}\right)\right|_{C}$ is ample, and

(5) if we write $\left.\left(K_{\tilde{S}}+B_{\tilde{S}}^{*}\right)\right|_{C}=K_{C}+\Theta$, there exists a divisor $\Theta^{\prime} \geq \Theta$ on $C$ whose coefficients belong to $D(J)$ and such that $\left(C, \Theta^{\prime}\right)$ is $\log$ Fano.

By Corollary 4.1, it follows that if $p$ is sufficiently large, depending only on $J$ (and therefore depending only on $I)$, then $\left(C, \Theta^{\prime}\right)$ is globally $F$-regular. Thus, $(C, \Theta)$ is also globally $F$-regular, and the statement follows again by Proposition 2.11

\section{ACKNOWLEDGEMENT}

The authors would like to thank H. Tanaka and Y. Prokhorov for many useful discussions. They would also like to thank the referee for carefully reading the paper and for many helful suggestions.

\section{REFERENCES}

[1] Bhargav Bhatt and Anurag K. Singh, The F-pure threshold of a Calabi-Yau hypersurface, Math. Ann. 362 (2015), no. 1-2, 551-567, DOI 10.1007/s00208-014-1129-0. MR3343889

[2] Caucher Birkar, Existence of flips and minimal models for 3-folds in char p, (2013), arXiv:1311.3098.

[3] Omprokash Das, On strongly F-regular inversion of adjunction, J. Algebra 434 (2015), 207226, DOI 10.1016/j.jalgebra.2015.03.025. MR3342393

[4] Christopher Hacon and Chenyang $\mathrm{Xu}$, On the three dimensional minimal model program in positive characteristic, (2013), arXiv:1302.0298.

[5] Nobuo Hara, Classification of two-dimensional F-regular and F-pure singularities, Adv. Math. 133 (1998), no. 1, 33-53, DOI 10.1006/aima.1997.1682. MR.1492785 (99a:14048)

[6] Nobuo Hara, F-pure thresholds and F-jumping exponents in dimension two, with an appendix by Paul Monsky, Math. Res. Lett. 13 (2006), no. 5-6, 747-760, DOI 10.4310/MRL.2006.v13.n5.a6. MR.2280772(2007m:14032)

[7] Nobuo Hara and Kei-Ichi Watanabe, F-regular and F-pure rings vs. log terminal and log canonical singularities, J. Algebraic Geom. 11 (2002), no. 2, 363-392, DOI 10.1090/S10563911-01-00306-X. MR.1874118 (2002k:13009)

[8] Nobuo Hara and Ken-Ichi Yoshida, A generalization of tight closure and multiplier ideals, Trans. Amer. Math. Soc. 355 (2003), no. 8, 3143-3174 (electronic), DOI 10.1090/S0002-994703-03285-9. MR.1974679 (2004i:13003)

[9] János Kollár, Singularities of the minimal model program, with the collaboration of Sándor Kovács, Cambridge Tracts in Mathematics, vol. 200, Cambridge University Press, Cambridge, 2013. MR3057950 
[10] János Kollár and Shigefumi Mori, Birational geometry of algebraic varieties, with the collaboration of C. H. Clemens and A. Corti; translated from the 1998 Japanese original. Cambridge Tracts in Mathematics, vol. 134, Cambridge University Press, Cambridge, 1998. MR 1658959 (2000b:14018)

[11] James McKernan and Yuri Prokhorov, Threefold thresholds, Manuscripta Math. 114 (2004), no. 3, 281-304, DOI 10.1007/s00229-004-0457-x. MR2075967 (2005g:14036)

[12] Paul Monsky, Mason's theorem and syzygy gaps, J. Algebra 303 (2006), no. 1, 373-381, DOI 10.1016/j.jalgebra.2005.10.012. MR.2253667(2007e:13024)

[13] Yu. G. Prokhorov and V. V. Shokurov, Towards the second main theorem on complements, J. Algebraic Geom. 18 (2009), no. 1, 151-199, DOI 10.1090/S1056-3911-08-00498-0. MR2448282 (2009i:14007)

[14] Yuri G. Prokhorov, Lectures on complements on log surfaces, MSJ Memoirs, vol. 10, Mathematical Society of Japan, Tokyo, 2001. MR.1830440 (2002e:14027)

[15] Karl Schwede, F-adjunction, Algebra Number Theory 3 (2009), no. 8, 907-950, DOI 10.2140/ant.2009.3.907. MR2587408(2011b:14006)

[16] Karl Schwede and Karen E. Smith, Globally F-regular and log Fano varieties, Adv. Math. 224 (2010), no. 3, 863-894, DOI 10.1016/j.aim.2009.12.020. MR2628797 (2011e:14076)

[17] Karl Schwede, Kevin Tucker, and Wenliang Zhang, Test ideals via a single alteration and discreteness and rationality of F-jumping numbers, Math. Res. Lett. 19 (2012), no. 1, 191197, DOI 10.4310/MRL.2012.v19.n1.a15. MR2923185

[18] Karen E. Smith, Globally F-regular varieties: applications to vanishing theorems for quotients of Fano varieties, Dedicated to William Fulton on the occasion of his 60th birthday, Michigan Math. J. 48 (2000), 553-572, DOI 10.1307/mmj/1030132733. MR.1786505 (2001k:13007)

[19] Shunsuke Takagi, An interpretation of multiplier ideals via tight closure, J. Algebraic Geom. 13 (2004), no. 2, 393-415, DOI 10.1090/S1056-3911-03-00366-7. MR2047704 (2005c:13002)

[20] Osamu Fujino and Hiromu Tanaka, On log surfaces, Proc. Japan Acad. Ser. A Math. Sci. 88 (2012), no. 8, 109-114, DOI 10.3792/pjaa.88.109. MR2989060

[21] Chenyang Xu, Finiteness of algebraic fundamental groups, Compos. Math. 150 (2014), no. 3, 409-414, DOI 10.1112/S0010437X13007562. MR3187625

Department of Mathematics, Imperial College London, London SW7 2AZ, United KINGDOM

E-mail address: p.cascini@imperial.ac.uk

Graduate School of Mathematical Sciences, University of Tokyo, 3-8-1 Komaba, Meguro-ku, Tokyo 153-8914, Japan - And - Department of Mathematics, Imperial ColLege London, 180 QueEn's Gate, London SW7 2AZ, United Kingdom

E-mail address: gongyo@ms.u-tokyo.ac.jp

E-mail address: y.gongyo@imperial.ac.uk

Department of Mathematics, Pennsylvania State University, University Park, PennSYLVANIA 16802

E-mail address: schwede@math.psu.edu

Current address: Department of Mathematics, University of Utah, 155 S 1400 E Room 233,

Salt Lake City, Utah 84112

E-mail address: schwede@math.utah.edu 